\title{
Bifidobacterium longum/Lactobacillus acidophilus/Enterococcus faecalis Probiotic Supplement
}

\author{
National Cancer Institute
}

\section{Source}

Nationa/ Cancer Institute. Bifidobacterium longum/Lactobacillus

acidophilus/Enterococcus faecalis Probiotic Supplement. NCI Thesaurus. Code C162855.

An orally bioavailable, probiotic supplement containing the microorganisms

Bifidobacterium longum (B. longum), Lactobacillus acidophilus (L. acidophilus) and Enterococcus faecalis (E. faecalis), with potential immunomodulating, anti-diarrheal and mucosal protective activities. Upon oral ingestion, the naturally-occurring bacterial components in B. longum/L. acidophilus/E. faecalis probiotic supplement may improve digestion and help to maintain adequate colonization of the gastrointestinal (Gl) tract by modulating the composition of the normal microflora. They produce lactic acid, hydrogen peroxide and other substances during fermentation, thereby creating an acidic environment that is unfavorable for pathogens. During colonization in the Gl tract, the bacteria may also form a protective intestinal barrier, which may prevent damage to the mucosal epithelium, maintain the integ rity of the epithelial barrier from potential damage by toxins and certain chemicals, and reduce the potential for attachment of pathogens. This further protects against bacterial translocation and infection. In addition, this agent may reduce the secretion of pro-inflammatory cytokines and may potentiate natural and acquired immunity. Altogether, this may increase immunity, prevent Gl mucosal damage, malabsorption, inflammation, and reduce diarrhea. 